\title{
Ajuste de curvas de crescimento em codornas de corte por inferência Bayesiana
}

\author{
Adjustment of growth curves in cutting quails by Bayesian inference \\ Ajuste de las curvas de crecimiento en codornices de corte por inferencia bayesiana
}

\author{
Ana Paula Silva Ton \\ ORCID: https://orcid.org/0000-0002-5826-1874 \\ Universidade Federal de Mato Grosso, Brasil \\ E-mail: anatonn4@hotmail.com \\ Monyka Marianna Massolini Laureano \\ ORCID: https://orcid.org/0000-0002-1702-6413 \\ Universidade Federal de Mato Grosso, Brasil \\ E-mail: monyka.laureano@gmail.com \\ Simone Inoe Araújo \\ ORCID: http://orcid.org/0000-0002-8125-6774 \\ Universidade Federal de Mato Grosso, Brasil \\ E-mail: araujoinoe@bol.com.br \\ Flávio Luiz de Menezes \\ ORCID: https://orcid.org/0000-0002-4009-9338 \\ Universidade Federal de Mato Grosso, Brasil \\ E-mail: flm.zootecnista@gmail.com \\ Cláudio Vieira de Araújo \\ ORCID: https://orcid.org/0000-0001-9378-7348 \\ Universidade Federal de Mato Grosso, Brasil \\ E-mail: cvaufmt@gmail.com
}

\begin{abstract}
Resumo
Objetivou-se comparar curvas de crescimento em codornas de corte (Coturnix coturnix coturnix), entre sexo e dietas com diferentes níveis de energia metabolizável (EM), por meio de modelos de regressão não lineares sob inferência Bayesiana. Utilizaram-se registros de pesos corporais referentes à 579 machos e 581 fêmeas, pesadas ao nascimento, aos 7, 14, 21, 35 e 42 dias de idade. As dietas foram compostas por três diferentes níveis de energia metabolizável, sendo 2750; 2950 e $3150 \mathrm{kcal}$ de EM/kg1. Os modelos avaliados empregaram as funções de Gompertz, Logística, Richards e Von Bertalanffy, considerando-se o parâmetro relacionado à taxa de maturidade (K) como aleatório. Foram geradas cadeias de Markov, via amostrador de Gibbs, adotando-se o Critério de Deviance na escolha do modelo mais adequado. Em ambos os sexos, a função de Gompertz apresentou ajuste significativamente superior de acordo com o critério avaliado, e menor estimativa de variância residual, indicativo de maior precisão na predição do peso corporal dos animais. Foi observada taxa de crescimento absoluto distintas entre os sexos, dessa forma a descrição de curvas de crescimento deve ser realizada de forma individualizada. As análises indicaram que o nível de $2750 \mathrm{kcal} / \mathrm{kg}$ de EM na dieta promove o crescimento de forma similar aos outros níveis na fase de 1 a 14 dias de idade em machos e de 1 a 21 dias de idade em fêmeas. Após estas idades, observou-se melhor desempenho utilizando-se o nível de $3150 \mathrm{kcal} / \mathrm{kg}$ de EM para machos e níveis de 2950 e $3150 \mathrm{kcal} / \mathrm{kg}$ para fêmeas.
\end{abstract}

Palavras-chave: Desenvolvimento ponderal; Energia metabolizável; Inferência bayesiana, Modelo de Gompertz; Taxa de crescimento absoluto.

\begin{abstract}
The aim was to compare growth curves in meat quails (Coturnix coturnix coturnix), between gender and diets with different levels of metabolizable energy (ME), using non-linear regression models under Bayesian inference. Body weight records were used for 579 males and 581 females, weighed at birth, at 7, 14, 21, 35 and 42 days of age. The diets were composed by three different levels of metabolizable energy, being 2750; 2950 and $3150 \mathrm{kcal} \mathrm{of} \mathrm{EM} \mathrm{/} \mathrm{kg.}$ The models used the functions of Gompertz, Logística, Richards and Von Bertalanffy, considering the parameter related to the maturity rate $(\mathrm{K})$ as random. Markov chains were generated via Gibbs sampler, adopting the Deviance Criterion in choosing the most appropriate model. In both genders. The Gompertz function showed a significantly higher adjustment according to the evaluated criterion, and a lower residual variance estimate, indicating greater precision in the prediction of the animals' body weight. Different absolute growth rates were observed between the genders, so the description of growth curves must be carried by gender. The analyzes indicated that the level of 2750 $\mathrm{kcal} / \mathrm{kg}$ of ME in the diet promotes growth in a similar way to the other levels in the phase from 1 to 14 days of age in males and from 1 to 21 days of age in females. After these ages, a better performance was observed using the level of $3150 \mathrm{kcal} / \mathrm{kg}$ of EM for males and levels of 2950 and $3150 \mathrm{kcal} / \mathrm{kg}$ for females.
\end{abstract}


Keywords: Ponderal development; Metabolizable energy; Bayesian inference, Gompertz model; Absolute growth rate.

\section{Resumen}

El objetivo fue comparar curvas de crecimiento en codornices de carne (Coturnix coturnix coturnix), entre sexo y dietas con diferentes niveles de energía metabolizable, utilizando modelos de regresión no lineal bajo inferencia bayesiana. Se utilizaron registros de peso corporal para 579 machoss y 581 hembras, pesados al nacer, a los 7, 14, 21, 35 y 42 días de edad. Las dietas estaban compuestas por tres niveles diferentes de energía metabolizable (EM), siendo 2750; 2950 y $3150 \mathrm{kcal}$ de EM / kg1. Los modelos evaluados utilizaron las funciones de Gompertz, Logística, Richards y Von Bertalanffy, considerando aleatorio el parámetro relacionado con la tasa de madurez (K). Se generaron cadenas de Markov, a través del muestreador de Gibbs, adoptando el Criterio de Desviation al elegir el modelo más apropiado. En ambos sexos, la función de Gompertz mostró un ajuste significativamente mayor según el criterio evaluado, y una estimación de la varianza residual menor, lo que indica una mayor precisión en la predicción del peso corporal de los animales. Se observaron diferentes tasas de crecimiento absoluto entre sexos, por lo que la descripción de las curvas de crecimiento debe realizarse de forma individual. Los análisis indicaron que el nivel de $2750 \mathrm{kcal} / \mathrm{kg}$ de EM en la dieta promueve el crecimiento de manera similar a los otros niveles en la fase de 1 a 14 días de edad en machos y de 1 a 21 días de edad en hembras. Luego de estas edades, se observó un mejor desempeño utilizando el nivel de $3150 \mathrm{kcal} / \mathrm{kg}$ de EM para machos y niveles de 2950 y $3150 \mathrm{kcal} / \mathrm{kg}$ para hembras.

Palabras clave: Desarrollo ponderal; Energía metabolizable; Inferencia bayesiana; Modelo de Gompertz; Tasa de crecimiento absoluta.

\section{Introdução}

Os modelos não lineares se projetam como eficientes instrumentos nas diferentes atividades relacionadas à produção animal, pois permitem descrever o desenvolvimento ponderal dos animais, em função da idade, utilizando-se de poucos parâmetros e, dessa forma, permitem alterações de taxa de crescimento durante a trajetória do desenvolvimento corporal, a qual são apontadas por meio de estimativas dos parâmetros da função não linear, bem como por meio de índices obtidos a partir dos mesmos.

Dessa forma, torna-se possível a comparação entre grupos de animais submetidos à diferentes classes de efeito sistêmicos, tais como, por exemplo, dietas, ambientes, linhagens e sexo. Nesse sentido, a trajetória da curva de crescimento dos animais pode ser utilizada para comparações entre dietas com diferentes níveis nutricionais. Visto a necessidade de elaboração de planos nutricionais que conduzam à redução de custo de produção.

Entre os nutrientes mais importantes na dieta, a energia metabólica relaciona-se intrinsicamente ao consumo de ração, bem como necessária para melhorias no desempenho produtivo e reprodutivos. Barreto et al. (2007) relatam que, especialmente, em codornas, cujo crescimento acontece de forma acelerada, a energia é o ponto chave para redução de custos e para obtenção de alta eficiência de produção.

Quando ocorre aumento no nível energético da ração, o consumo voluntário diminui (Corrêa et al., 2007; Ton et al., 2011). Dessa forma, além da proteína dietética, as exigências nutricionais das aves devem ser expressas em relação ao nível de energia da dieta.

Pesquisadores afirmam que, em codornas, o consumo é regulado de acordo com a necessidade de energia e exigência nutricional, no entanto, outros fatores também podem influenciar o consumo, como a necessidade da ave, o peso corporal, a fase de crescimento e o ambiente de criação, entre outros fatores (Moura et al., 2010; Hurtado et al., 2014).

Todavia, comparações entre dietas com diferentes composições nutricionais por meio de funções não lineares, geralmente são obtidas utilizando-se estimadores de quadrados mínimos ou máxima verossimilhança restrita. Nessa situação, os contrastes obtidos por meio de teste de hipóteses envolvem desvios-padrão obtidos para médias desses efeitos sistêmicos que são assintóticos.

Rossi e Santos (2014) relatam que modelos de regressão não lineares com erros normais são usualmente aplicados em muitas áreas de estudo, porém em muitas situações, devido à falta de simetria, a suposição de normalidade não se aplica. 
Nesse contexto, enquanto na abordagem de inferência frequentista, o conjunto de parâmetros a ser estimado é considerado uma quantidade constante e, portanto, sem uma distribuição de probabilidade associado ao mesmo. Na inferência Bayesiana, esse mesmo conjunto de parâmetros é considerado uma quantidade aleatória, e, consequentemente, possui uma distribuição de probabilidade (priori).

Nesse sentido, a abordagem bayesiana evita inferências assintóticas e com o uso de informações à priori sobre os parâmetros de interesse, resultando em distribuição a posteriori mais consistente e com aumento de acurácia (Spiegelhalter et al 2002).

Objetivou-se, comparar trajetórias de curvas de crescimento de codornas de corte entre sexos e dietas com diferentes níveis de energia metabolizável, por meio de diferentes modelos de regressão não lineares, utilizando-se de inferência Bayesiana.

\section{Metodologia}

\section{Animais}

Utilizaram-se informações referentes a registros de peso corporais de codornas de corte (Coturnix coturnix coturnix) pesadas ao nascimento, aos 7, 14, 21, 35 e 42 dias de idade, referentes à 579 machos e 581 fêmeas. Os animais foram provenientes de um experimento realizado no Laboratório de Nutrição de Monogástricos, pertencente à Universidade Federal de Mato Grosso, Campus de Sinop, em Mato grosso, MT

As aves foram alimentadas, com dietas compostas por três diferentes níveis de energia metabolizável iguais a 2750; 2950 e $3150 \mathrm{kcal}$ de EM/kg-1. A composição bromatológica das dietas são descritas na Tabela 1 e a estatística descritiva dos pesos corporais das aves em cada idade avaliada e submetidas às diferentes dietas são exibidos na Tabela 2. 
Tabela 1. Composições percentual e nutricional das rações experimentais com níveis de energia metabolizável para codornas de corte.

\begin{tabular}{|c|c|c|c|}
\hline \multirow[b]{2}{*}{ Ingredientes $(\%))$} & \multicolumn{3}{|c|}{ Níveis de energia metabolizável (kcal/kg) } \\
\hline & 2750 & 2950 & 3150 \\
\hline Milho grão $(7,86 \%)$ & 53,680 & 53,675 & 49,030 \\
\hline Farelo de soja $(45 \%)$ & 39,870 & 41,050 & 41,842 \\
\hline Casca de soja & 3,500 & 0,000 & 0,000 \\
\hline Óleo de soja & 0,000 & 2,300 & 6,150 \\
\hline Fosfato bicálcico & 0,960 & 0,973 & 0,980 \\
\hline Calcário calcítico & 0,929 & 0,960 & 0,950 \\
\hline DL-Metionina & 0,281 & 0,273 & 0,278 \\
\hline L-Lisina $\mathrm{HCl}$ & 0,010 & 0,000 & 0,000 \\
\hline Cloreto de sódio & 0,370 & 0,369 & 0,370 \\
\hline Suplemento mineral + vitamínico ${ }^{1}$ & 0,400 & 0,400 & 0,400 \\
\hline Total (kg) & 100,00 & 100,00 & 100,00 \\
\hline \multicolumn{4}{|l|}{ Composição nutricional } \\
\hline Energia metabolizável (kcal/kg) & 2750 & 2950 & 3150 \\
\hline Lisina digestível (\%) & 1,14 & 1,14 & 1,14 \\
\hline Proteína bruta (\%) & 23,00 & 23,00 & 23,00 \\
\hline Fibra bruta $(\%)$ & 4,02 & 2,92 & 2,88 \\
\hline Met+cist digestível (\%) & 0,89 & 0,89 & 0,89 \\
\hline Cálcio (\%) & 0,75 & 0,75 & 0,75 \\
\hline Fósforo disponível (\%) & 0,29 & 0,29 & 0,29 \\
\hline Sódio (\%) & 0,16 & 0,16 & 0,16 \\
\hline
\end{tabular}

${ }^{1}$ Suplementação vitamínica/mineral (níveis de garantia por kg de ração); Vit. A - 8.100 UI; Vit. D3 - 3.000 UI; Vit. E - 6 mg/kg; Vit. B1 $1,05 \mathrm{mg} / \mathrm{kg}$; Vit. B2 - $3 \mathrm{mg} / \mathrm{kg}$; Vit. B6 - 1,8 mg/kg; Vit. B12 - $9 \mu \mathrm{g} / \mathrm{kg} ;$ Vit. K3 - 1,2 mg/kg; Ácido fólico - 0,3 mg/kg; Biotina - 0,03 $\mathrm{mg} / \mathrm{kg}$; Pantotenato de Cálcio - 6,6 mg/kg; Niacina - $18 \mathrm{mg} / \mathrm{kg}$; Cloreto de Colina - $78 \mathrm{mg} / \mathrm{kg}$; Óxido de Zinco - $90 \mathrm{mg} / \mathrm{kg}$; Sulfato de Ferro - $54 \mathrm{mg} / \mathrm{kg}$; Óxido de Manganês - $105 \mathrm{mg} / \mathrm{kg}$; Sulfato de Cobre - 8,1 mg/kg; Sulfato de Cobalto - 0,21 mg/kg; Iodato de cálcio - 0,78 $\mathrm{mg} / \mathrm{kg}$; Selenito de sódio - 0,3 mg/kg; BHT 3,6 mg/kg; Etoxiquin 1,5 mg/kg; Caulim. Fonte: Autores. 
Tabela 2. Número de observações (N), média e desvio-padrão (DP) para o peso corporal (em gramas) de codornas de corte em diferentes idades (dias).

\begin{tabular}{lcccccc}
\hline EM (kcal/kg) & \multicolumn{7}{c}{2.750} & & 2.950 & & 3.150 \\
\hline Machos & $\mathrm{N}$ & Média (DP) & $\mathrm{N}$ & Média (DP) & $\mathrm{N}$ & Média (DP) \\
\hline Idade & 207 & $10,25(0,98)$ & 181 & $10,15(1,04)$ & 191 & $10,06(1,00)$ \\
\hline 1 & 206 & $29,05(6,78)$ & 176 & $29,16(6,54)$ & 189 & $28,75(6,64)$ \\
7 & 206 & $74,69(13,16)$ & 180 & $71,11(14,43)$ & 189 & $73,78(13,54)$ \\
14 & 207 & $125,13(23,11)$ & 180 & $116,60(29,17)$ & 188 & $124,16(26,85)$ \\
21 & 202 & $182,95(21,01)$ & 178 & $175,46(25,74)$ & 190 & $180,47(27,74)$ \\
28 & 199 & $224,59(20,82)$ & 174 & $219,43(24,72)$ & 188 & $222,86(28,74)$ \\
35 & 182 & $244,63(22,27)$ & 154 & $245,23(22,53)$ & 169 & $248,05(26,77)$ \\
42 & & & & & & \\
\hline Fêmeas & $\mathrm{N}$ & Média (DP) & $\mathrm{N}$ & Média (DP) & $\mathrm{N}$ & Média (DP) \\
\hline Idade & 187 & $10,40(1,07)$ & 184 & $10,27(1,10)$ & 210 & $10,30(0,95)$ \\
\hline 1 & 185 & $30,32(7,16)$ & 182 & $31,65(6,89)$ & 208 & $29,99(7,17)$ \\
7 & 186 & $79,30(14,42)$ & 183 & $78,33(14,44)$ & 209 & $78,44(15,24)$ \\
14 & 184 & $132,82(25,93)$ & 183 & $130,12(28,72)$ & 210 & $132,84(30,48)$ \\
21 & 182 & $193,46(23,72)$ & 179 & $191,38(26,66)$ & 209 & $190,43(31,81)$ \\
28 & 171 & $241,60(22,10)$ & 171 & $239,07(28,16)$ & 197 & $239,60(37,00)$ \\
35 & 165 & $277,85(26,53)$ & 161 & $283,31(30,59)$ & 178 & $287,55(35,19)$ \\
42 & & & & &
\end{tabular}

Fonte: Autores.

\section{Modelos}

Os registros de pesos corporais, em gramas, foram regredidos em função da idade, em dias, por meio de modelos não lineares que empregaram as funções de Gompertz: 『『y ${ }_{-} \mathrm{t}=\mathrm{A}+\mathrm{e} \rrbracket \wedge\left(\mathrm{e}^{\wedge}(-\mathrm{B}(-(\mathrm{k}+\mathrm{k} 1) \mathrm{t}))\right)$; Logística: 『y $\rrbracket \_\mathrm{t}=\mathrm{A}+(1+\mathrm{Be}-$ $(\mathrm{k}+\mathrm{k} 1) \mathrm{t})-1$; Richards: $\llbracket \mathrm{y} \rrbracket \_\mathrm{t}=\mathrm{A}+(1-\mathrm{Be}-(\mathrm{k}+\mathrm{k} 1) \mathrm{t}) \mathrm{m}$ e Von Bertalanffy: 『y $\mathrm{y}_{-} \mathrm{t}=\mathrm{A}+(1-\mathrm{Be}-(\mathrm{k}+\mathrm{k} 1) \mathrm{t}) 3$, em que Yt peso corporal da ave (em gramas) na idade " $t$ " (em dias); em que "A" é o parâmetro de peso assintótico à maturidade da ave; "B" é uma constante de integração, sem interpretação biológica; "K" é um parâmetro relacionado a taxa de maturidade e "m" parâmetro relacionado ao formato da curva.

As análises foram processadas considerando-se em todos os modelos não lineares o parâmetro relacionado à taxa de maturidade (K) como sendo aleatório.

Os modelos foram processados utilizando inferência Bayesiana adotando distribuição a priori inversa de Gamma para os parâmetros do modelo e para os componentes de variância associados ao resíduo (S_e ${ }^{\wedge} 2$ ) e ao parâmetro K (S_k^2) adotouse prioris conjugadas Normal.

Foram geradas cadeias de Markov, por meio de amostrador de Gibbs, de tamanho total de 500000 ciclos, com burn in de 50.000 ciclos e thin in igual a 20 ciclos. O diagnóstico das cadeias de Markov foi realizado por meio do Critério de Geweke, adotando-se o nível de 5\% de significância.

Utilizou-se o Critério de Deviance (DIC) para a escolha do modelo de regressão não linear mais adequado em descrever o comportamento da curva de crescimento dos animais. 
De posse da melhor função empregada no modelo de regressão não linear, o mesmo foi utilizado na regressão do peso corporal em função da idade por sexo e, ainda por nível de energia metabolizável em cada sexo.

Comparações entre modelos em diferentes sexos e entre diferentes níveis energéticos em cada sexo, foram obtidas por meio de contrastes aos pares envolvendo os valores de Critério de Deviance (DIC) dos modelos. Rossi et al. (2017) discutem que quando a diferença entre valores de DIC entre dois modelos é menor que cinco, então não há diferenças entre ambos, quando essa diferença estiver entre cinco e dez, então os modelos podem ser considerados diferentes e, ainda, com diferenças maiores que dez, as diferenças são altamente significativas.

Ainda, comparações entre médias posteriores dos parâmetros da função obtidos entre sexo e entre níveis de energia metabolizável em cada sexo, foram realizadas por meio dos intervalos de credibilidade, adotando-se o nível de 95\% de certeza, obtidos por meio das cadeias de Markov.

\section{Resultados e Discussão}

Em todas as análises os parâmetros dos modelos utilizados não apresentaram o Critério de Geweke significativo ( $>0,05)$, indicando que os tamanhos das cadeias de Markov, geradas pelo amostrador de Gibbs foram satisfatórios e as cadeias estacionárias.

Em ambos os sexos o modelo que empregou a função de Gompertz apresentou o menor valor de DIC e, quando realizado os contrastes aos pares dos valores de DIC entre todos os modelos, em todas as situações os valores apresentaram-se maiores que 10, indicando diferenças significativas de ajustes entre todos os modelos (Tabela 3).

Tabela 3. Modelos de regressão não linear empregados na predição do peso corporal de codornas de corte $\left(\hat{y}_{i}\right)$, Critério de informação de Deviance (DIC), médias posteriores de variâncias residual (var(e)) e do parâmetro K (var(K)).

\begin{tabular}{llccc}
\hline Machos & \multicolumn{1}{c}{ Modelos } & DIC & $\operatorname{var}(\mathrm{e})$ & $\operatorname{var}(\mathrm{K})$ \\
\hline Gompertz & $\hat{y}_{\mathrm{i}}=305,0(\exp (-3,9622 \exp (-0,0720 \mathrm{X})))$ & 29797,65 & 98,54 & 0,00011 \\
Logístico & $\hat{y}_{\mathrm{i}}=267,1(1+19,24986 \exp (-0,1335 \mathrm{X}))^{-1}$ & 29999,41 & 103,9 & 0,00031 \\
Richards & $\hat{y}_{\mathrm{i}}=393,2(1-1,0152 \exp (-0,0248 \mathrm{X}))^{1,1352}$ & 34436,12 & 321,2 & 0,00005 \\
Von Bertalanffy & $\hat{y}_{\mathrm{i}}=337,1(1-0,8182 \exp (-0,0519 \mathrm{X}))^{3}$ & 30334,56 & 112,9 & 0,00008 \\
\hline Fêmeas & & & & \\
\hline Gompertz & $\hat{y}_{\mathrm{i}}=375,5(\exp (-3,7753 \exp (-0,0620 \mathrm{X})))$ & 29777,82 & 100,1 & 0,00009 \\
Logístico & $\hat{y}_{\mathrm{i}}=317,5(1+18,5046 \exp (-0,1207 \mathrm{X}))-1$ & 31013,46 & 137,6 & 0,00025 \\
Richards & $\hat{y}_{\mathrm{i}}=400,1(1-1,0160 \exp (-0,0277 \mathrm{X}))^{1,1533}$ & 36043,57 & 498,6 & 0,00005 \\
Von Bertalanffy & $\hat{y}_{\mathrm{i}}=449,3(1-0,7770 \exp (-0,0408))^{3}$ & 29834,42 & 101,5 & 0,00006 \\
\hline
\end{tabular}

Fonte: Autores.

Assim, verificou-se que o modelo de Gompertz apresentou menor estimativa de variância residual, implicando em maior precisão na predição do peso corporal dos animais. Dessa forma, o modelo que empregou a função não linear de Gompertz foi eleito como mais indicado para o ajuste na descrição de curvas de crescimento de codornas de corte, em ambos os sexos, seguido dos modelos Logístico, Von Bertalanffy e de Richards em machos, e dos modelos e de Von Bertalanffy, Logístico e de Richards, em fêmeas.

Rossi et al. (2017) avaliando o crescimento de codornas de corte (Coturnix coturnix coturnix) e de postura (Coturnix 
coturnix japonica), em um período similar ao verificado nesse estudo, também elegeram o modelo de Gompertz como mais indicado na descrição da curva de crescimento, independentemente da linhagem. Similarmente, Mota et al. (2015); Finco et al. (2016) e Firat et al. (2016) também elegeram a função de Gompertz como mais adequada na descrição de curvas de crescimento de codornas.

Drumond et al. (2013) verificaram que os modelos que mais se ajustaram para o crescimento de codornas foram o de Gompertz em machos e Logístico para fêmeas. Já Rocha-Silva et al. (2016) verificaram os modelos de Morgan Mercer Flodin e de Gompertz como os mais indicados para a predição de pesos corporais de codornas de corte machos e fêmeas, respectivamente.

Dessa forma, avaliando-se as estimativas dos parâmetros da função de Gompertz para descrição das curvas de crescimento por sexo (Tabela 4), verificou-se que as médias posteriores dos parâmetros da função se posicionaram em intervalos de credibilidades diferentes entre sexos. Desse modo, codornas de corte machos apesar de detentores de maior taxa de maturidade, ou velocidade de crescimento para se atingir o peso assintótico (parâmetro K), apresentaram menor peso assintótico ou peso à maturidade (parâmetro A) em relação às fêmeas. Assim, machos apresentam desenvolvimento ponderal mais acelerado e com menor ganho de peso corporal diário, em relação às fêmeas.

Tabela 4. Médias posteriores dos parâmetros da função de Gompertz com os respectivos desvios-padrão (DP) e limites inferior (li) e superior (ls) do intervalo de credibilidade, com 95\% de certeza, nos diferentes sexos.

\begin{tabular}{lcccc}
\hline & & \multicolumn{2}{c}{ Intervalo de credibilidade } \\
\hline Parâmetros & Média & DP & li & ls \\
\hline Machos & & & 302,6 & 307,5 \\
A & 305,0 & 1,2779 & 3,9147 & 4,0108 \\
B & 3,962 & 0,0246 & 0,0706 & 0,0733 \\
K & 0,072 & 0,00068 & & 375,8 \\
Fêmeas & & & 375,3 & 3,728 \\
A & 377,5 & 0,1371 & 3,7719 & 0,0628 \\
B & 3,7753 & 0,00177 & 0,0612 & \\
K & 0,062 & 0,000415 & . & \\
\hline
\end{tabular}

$\mathrm{A}=$ parâmetro de peso assintótico à maturidade da ave; $\mathrm{B}=$ constante de integração; $\mathrm{K}$ = parâmetro relacionado a taxa de maturidade. Fonte: Autores.

Rajini e Narahari (1998) avaliando requerimento nutricionais em dietas para codornas, verificaram diferenças entre sexos e apontaram o maior peso adulto em fêmeas devido ao desenvolvimento do aparelho reprodutivo e maior deposição de gordura. Grieser et al. (2018) corroboram com essa afirmativa atribuindo maior peso as codornas fêmeas devido ao aumento dos ovários e órgãos internos como fígado, proventrículo e moela, além da deposição corporal. Enquanto as codornas machos têm maior pelo de dorso e coração (Raji et al., 2015). Albino e Barreto (2003) afirmaram que em média o aparelho reprodutor representa $10 \%$ do peso corporal vivo de uma codorna adulta.

Avaliando os resultados obtidos por meio da função de Gompertz, verificou-se que machos apresentaram o ponto de inflexão, aproximadamente, aos 19 dias de idade e com peso corporal de 112 gramas e taxa de crescimento absoluta igual a 8,08 gramas/dia. Para as fêmeas, o ponto de inflexão ocorreu aproximadamente aos 21 dias com o peso corporal de 135 gramas e uma taxa de crescimento absoluta igual a 8,60 gramas/dia. Tal resultado denota a maior precocidade observada em machos (Figuras $1 \mathrm{e}$ 2). 
Figura 1. Curvas de crescimento com o peso corporal predito (gramas) e a taxa de crescimento absoluta (gramas/dia) para codornas de corte machos, segundo o modelo de Gompertz.

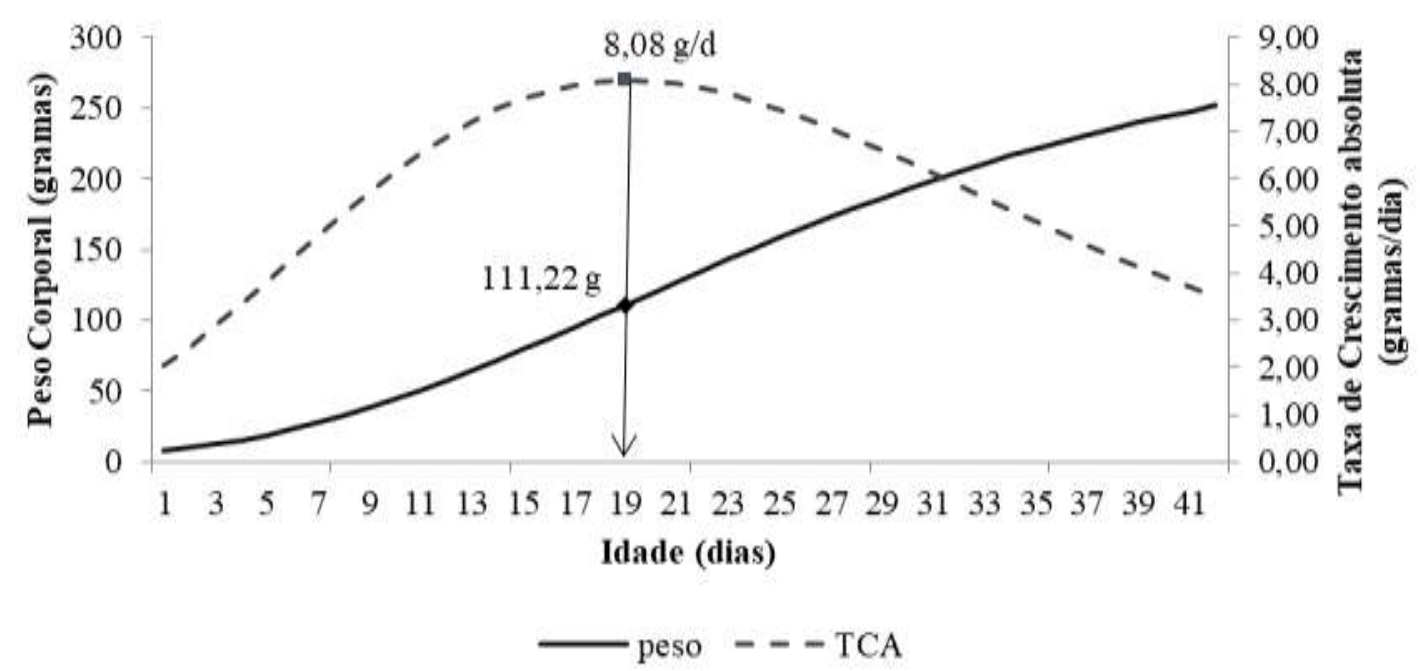

Fonte: Autores.

Figura 2. Curvas de crescimento com o peso corporal predito (gramas) e a taxa de crescimento absoluta (gramas/dia) para codornas de corte fêmeas, segundo o modelo de Gompertz.

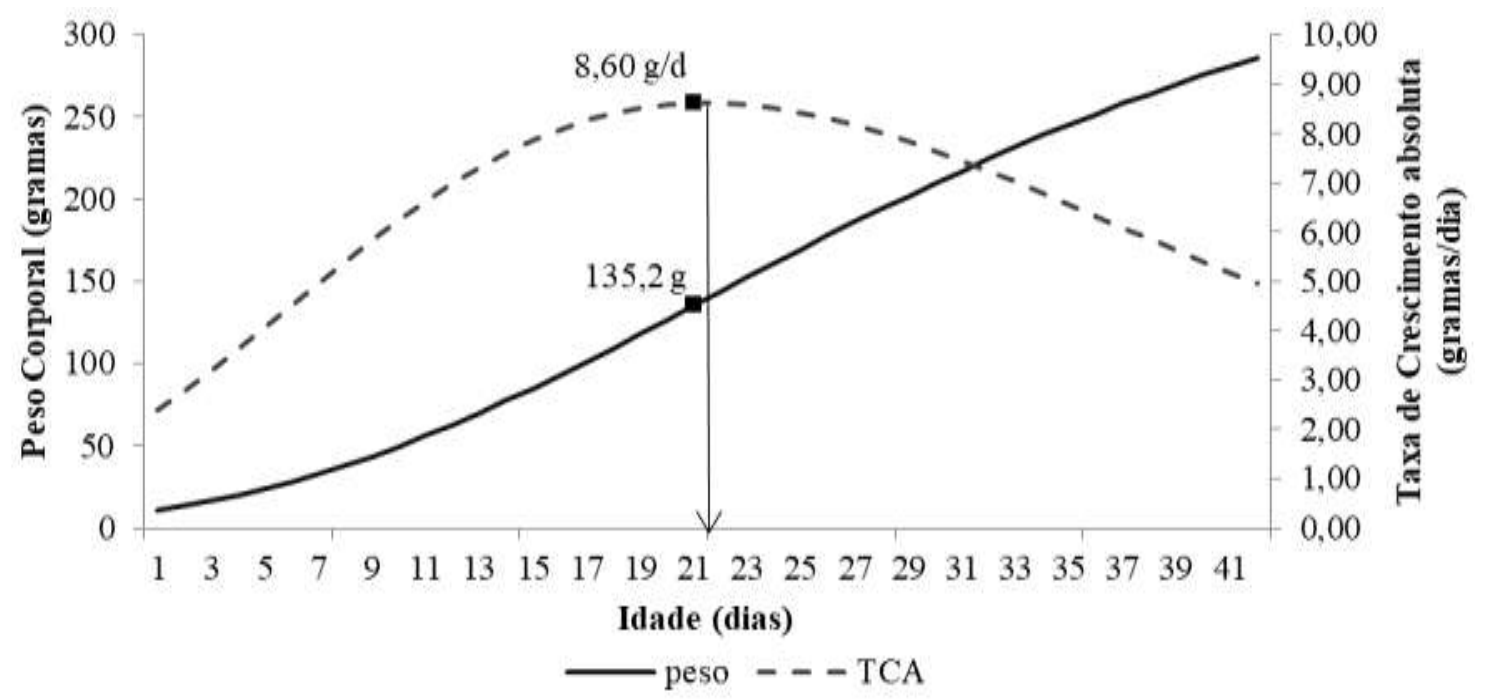

Fonte: Autores.

As codornas, sejam destinadas à produção de carne ou ovos, apresentam maturidade sexual precoce a qual está relacionada com a taxa de crescimento, ou seja, codornas menores têm taxa de crescimento superior e menor idade até a maturidade sexual, sendo assim, a precocidade no crescimento está relacionada a tempo que as codornas levam para tingir a maturidade sexual, sendo este parâmetro norteador na produção animal, além de demonstrar as diferentes necessidades nutricionais das codornas (Vieira et al., 2020).

Enquanto os machos apresentaram-se com predições de peso corporal de 7,64 ao início da trajetória e uma taxa de crescimento absoluta equivalente à 2,08 gramas/dia, as fêmeas apresentaram-se com valores equivalentes à 10,86 gramas e 2,38 gramas/dia, respectivamente. 
Aos 42 dias de idade, as codornas de corte machos e fêmeas apresentaram predições de peso corporal iguais à 251,57 e 285,51 gramas com taxas de crescimento absoluta equivalente à 3,49 e 4,94 gramas/dia, respectivamente.

Dessa forma, havendo diferenças fisiológicas para o crescimento entre machos e fêmeas, refletindo-se em pesos corporais distintos, bem como diferentes taxas de crescimento absoluta, deve-se manter a análise individualizada por sexo, mesmo que o mesmo modelo seja o mais ajustado para ambos os sexos, uma vez que os valores dos parâmetros estimados são diferentes.

Avaliando as curvas de crescimento por nível de energia metabolizável em cada sexo, separadamente, observou-se que os valores de DIC para os níveis de energia de 2750; 2950 e $3150 \mathrm{kcal} / \mathrm{kg}$ de EM, foram iguais à 10625,43; 9396,16 e 9771,99 para machos e 9261,86; 9524,80 e 10824,09, para fêmeas, respectivamente, indicando haver diferenças entre as curvas de crescimento obtidas entre diferentes níveis energéticos e em cada sexo. Dessa forma, ao avaliar os intervalos de credibilidade associados às médias posteriores (Tabela 5), verificou-se que em ambos os sexos, o peso corporal assintótico foi maior para o nível de energia metabolizável de $3150 \mathrm{kcal} / \mathrm{kg}$ na dieta, em relação ao nível $2750 \mathrm{kcal} / \mathrm{kg}$, com o valor intermediário de peso corporal assintótico ao nível de $2950 \mathrm{kcal} / \mathrm{kg}$ em machos e igual e superior aos valores obtidos nos níveis 3150 e $2750 \mathrm{kcal} / \mathrm{kg}$ na dieta, respectivamente, em fêmeas.

Tabela 5. Médias posteriores dos parâmetros da função de Gompertz com os respectivos desvios-padrão e limites inferior (li) e superior (ls) do intervalo de credibilidade, em cada sexo para os diferentes níveis energéticos das dietas.

\begin{tabular}{|c|c|c|c|c|c|c|c|c|}
\hline & & & \multicolumn{2}{|c|}{ Machos } & \multicolumn{4}{|c|}{ Fêmeas } \\
\hline \multicolumn{3}{|c|}{ EM $2750 \mathrm{kcal} / \mathrm{kg}$} & \multicolumn{2}{|c|}{ Intervalo de credibilidade } & \multirow[b]{2}{*}{ Média } & \multirow[b]{2}{*}{ Desvio Padrão } & \multicolumn{2}{|c|}{ Intervalo de credibilidade } \\
\hline Parâmetros & Média & Desvio Padrão & li & 1s & & & li & 1s \\
\hline A & 297,90 & 1,94 & 294,10 & 301,70 & 356,70 & 2,5071 & 351,80 & 361,70 \\
\hline B & 3,98 & 0,04 & 3,90 & 4,06 & 3,8891 & 0,0314 & 3,8225 & 3,9507 \\
\hline K & 0,0747 & 0,00126 & 0,0723 & 0,0773 & 0,0663 & 0,00119 & 0,0639 & 0,0686 \\
\hline \multicolumn{9}{|c|}{ EM $2950 \mathrm{kcal} / \mathrm{kg}$} \\
\hline A & 305,50 & 2,44 & 300,80 & 310,30 & 383,5 & 3,4588 & 377,30 & 390,10 \\
\hline B & 4,04 & 0,05 & 3,94 & 4,13 & 3,8518 & 0,0302 & 3,7957 & 3,9127 \\
\hline K & 0,0712 & 0,00141 & 0,0684 & 0,0739 & 0,0615 & 0,00119 & 0,0593 & 0,0640 \\
\hline \multicolumn{9}{|c|}{ EM $3150 \mathrm{kcal} / \mathrm{kg}$} \\
\hline A & 308,70 & 1,79 & 305,70 & 312,10 & 390,40 & 3,3096 & 383,90 & 396,90 \\
\hline B & 3,85 & 0,02 & 3,82 & 3,91 & 3,8528 & 0,0293 & 3,7943 & 3,9088 \\
\hline $\mathrm{K}$ & 0,0704 & 0,00116 & 0,0682 & 0,0727 & 0,0606 & 0,00115 & 0,0584 & 0,0629 \\
\hline
\end{tabular}

Fonte: Autores.

Ainda, em fêmeas, para o parâmetro K, associado à precocidade de crescimento, observou-se maior valor para o nível de $2750 \mathrm{kcal} / \mathrm{kg}$. quando comparado ao nível de $3150 \mathrm{kcal} / \mathrm{kg}$ na dieta, não havendo diferenças significativas para os machos entre os diferentes níveis energéticos.

Corrêa et al. (2017) avaliando exigências proteicas e energéticas em codornas, verificaram que o peso corporal aos 42 dias de idade, bem como, o ganho de peso e a conversão alimentar dos 7 aos 42 dias de idade, não foram influenciados pelos 
níveis de energia e de proteína nas dietas.

Avaliando o comportamento das curvas de crescimento em codornas de corte machos com os níveis energéticos iguais a 2750; 2950 e $3150 \mathrm{kcal} / \mathrm{kg}$, o ponto de inflexão ocorreu aos 18,5; 19;6 e 19,15 dias de idade com peso corporal correspondente à 109,6; 112,4 e 113,5 gramas de peso corporal, respectivamente. As taxas de crescimento absoluta obtidas nos pontos de inflexão das curvas foram iguais a 8,2; 7;9 e 8,4 gramas/dia, para os níveis energéticos iguais a 2750; 2950 e 3150 $\mathrm{kcal} / \mathrm{kg}$, respectivamente (Figura 3).

Figura 3. Taxa de Crescimento Absoluta em codornas de corte machos com os níveis de energia de 2750 (E1); 2950 (E2) e 3150 (E3) kcal/kg, utilizando a função de Gompertz.

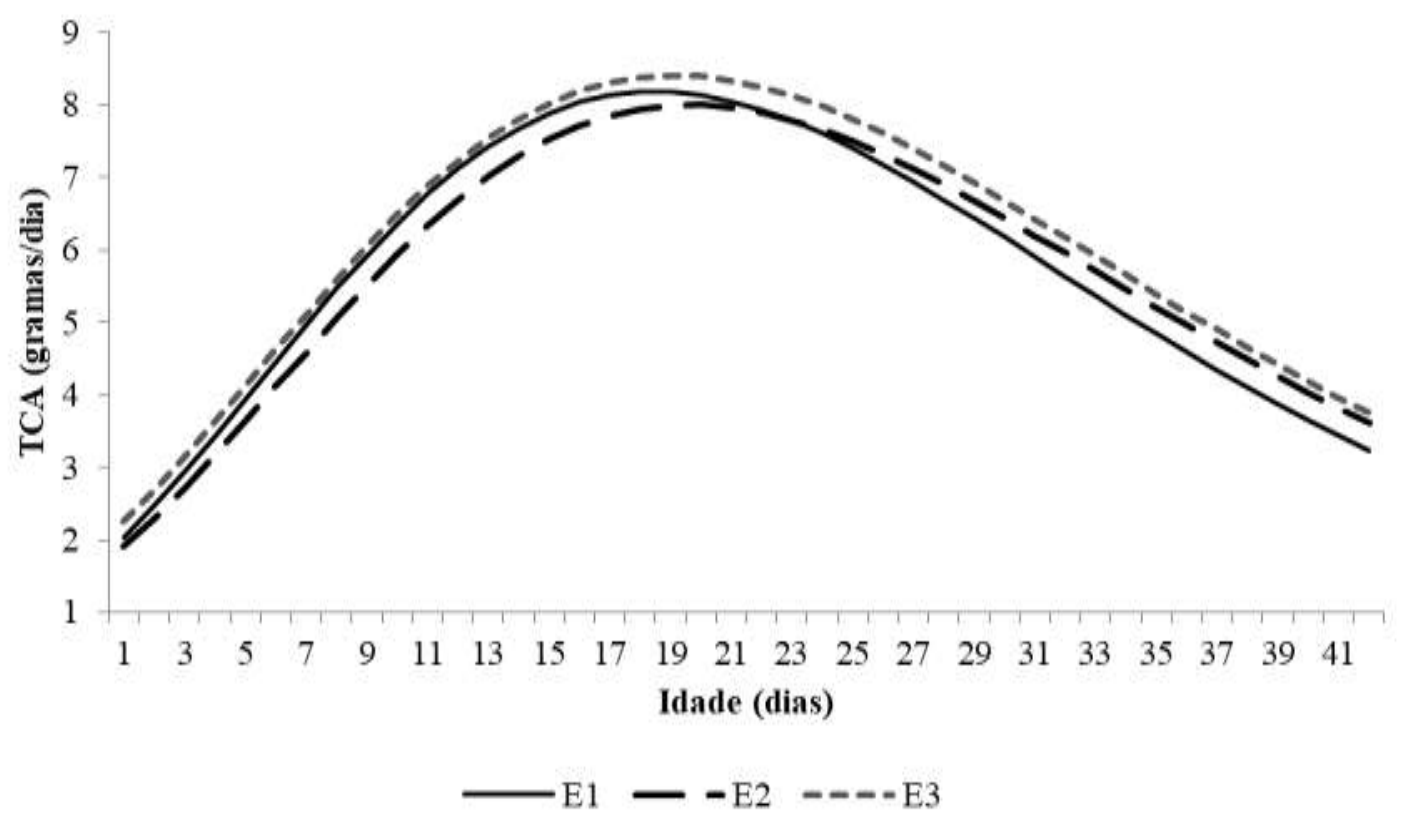

Fonte: Autores.

Em codornas de corte fêmeas os pontos de inflexão aos 20,5; 21,9 e 22,3 dias de idade com peso corporal correspondente à 131,2; 141,0 e 143,6 para os níveis energéticos iguais a 2750; 2950 e 3150 kcal $/ \mathrm{kg}$. Corresponderam a TCI iguais a 8,$6 ; 8,7$ e 8,8 , respectivamente (Figura 4). 
Figura 4. Taxa de Crescimento Absoluta em fêmeas para os diferentes para os níveis de energia de 2750 (E1); 2950 (E2) e 3150 (E3) kcal/kg, utilizando a função de Gompertz.

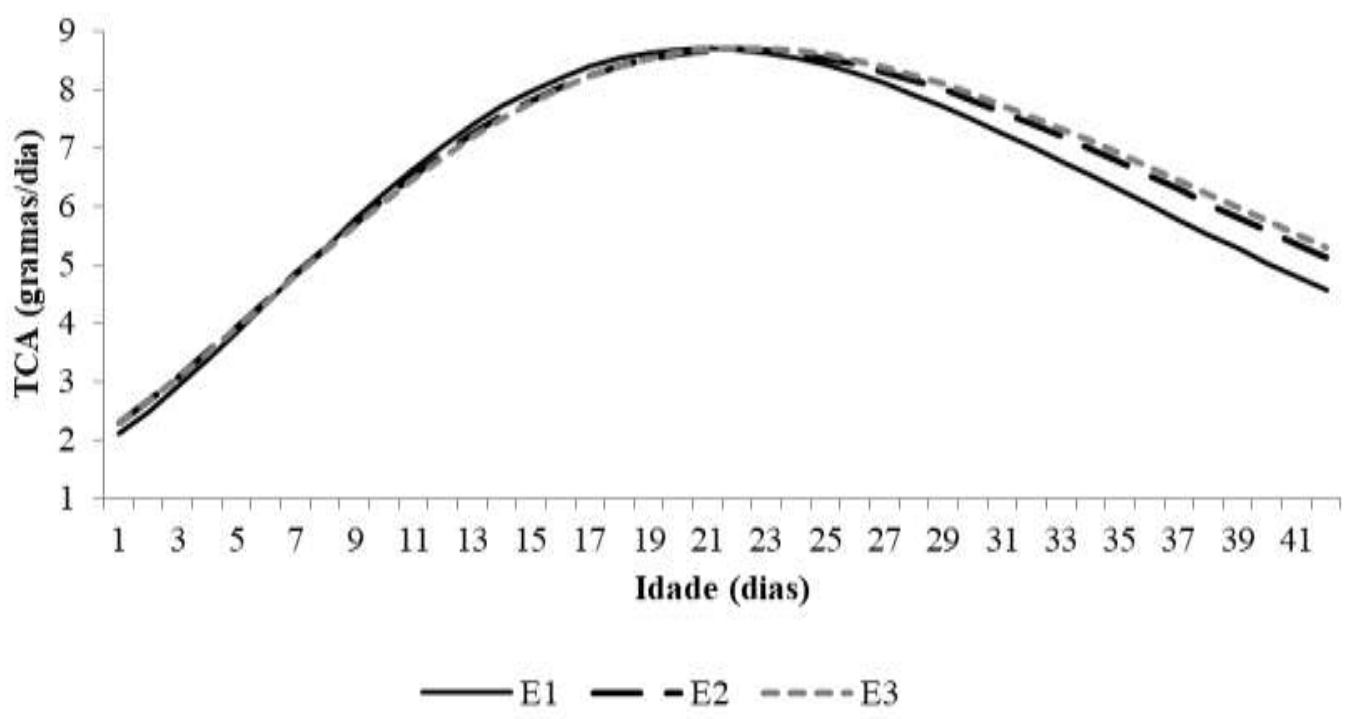

Fonte: Autores.

Portanto, codornas de corte tanto em machos quanto em fêmeas, o maior peso assintótico obtido no nível energético igual a $3150 \mathrm{kcal} / \mathrm{kg}$ em relação ao nível $2750 \mathrm{kcal}$., está associado ao maior crescimento dos animais após atingir o ponto de inflexão.

Dessa forma, verificou-se que o nível de $2750 \mathrm{kcal} / \mathrm{kg}$ de EM na dieta das codornas de corte, promove o crescimento de forma similar aos outros níveis na fase de 1 a 14 dias de idade em machos e de 1 a 21 dias de idade em fêmeas. Com melhores desempenhos, posteriormente, utilizando-se o nível de $3150 \mathrm{kcal} / \mathrm{kg}$ de EM para machos, enquanto em fêmeas os melhores desempenhos foram obtidos de forma similares nos níveis de 2950 e $3150 \mathrm{kcal} / \mathrm{kg}$, respectivamente (Figuras 3 e 4 ).

Dessa forma, a adoção de níveis diferentes de energia metabolizável ao longo da trajetória de crescimento ponderal dos animais permite promover ganhos de peso diário com incremento positivo na velocidade de crescimento e, consequentemente, redução do intervalo de tempo para atingir a idade adulta e, portanto, redução de custos com alimentação, além diminuir os intervalos de geração, e consequentemente, aumentar o ganho genético a cada geração em um programa de seleção genética.

\section{Conclusão}

O modelo de Gompertz é mais adequado na descrição das trajetórias de curvas de crescimento ponderal para codornas de corte machos e fêmeas, todavia, há diferenças fisiológicas que conduzem à diferentes características para o crescimento entre sexos e portanto, a descrição de curvas de crescimento ponderal deve ser realizada de forma distinta.

As codornas de corte devem ser criadas com dietas contendo o nível de energia metabolizável de $2750 \mathrm{kcal} / \mathrm{kg}$ na fase de 1 a 14 dias de idade para machos e de 1 a 21 dias de idade para fêmeas. Adotando-se, posteriormente, nas demais fases de crescimento, os níveis de energia metabolizável de 3150 e $2950 \mathrm{kcal} / \mathrm{kg}$ nas dietas, em machos e fêmeas, respectivamente.

Recomenda-se o uso da Inferência Bayesiana para obtenção de estimativas de parâmetros de curvas de crescimento em codornas, por permitir a escolha de distribuição à prior que resultam em distribuições posteriores mais precisas. 


\section{Agradecimentos}

À FAPEMAT - Fundação de Amparo à Pesquisa do Estado do Mato Grosso, O presente trabalho foi realizado com apoio da Coordenação de Aperfeiçoamento de Pessoal de Nível Superior - Brasil (CAPES) - Código de Financiamento 001 e ao Conselho Nacional de Desenvolvimento Científico e Tecnológico (CNPq) pelo auxílio financeiro na realização do projeto de pesquisa e pela concessão de bolsas de Iniciação Científica.

\section{Referências}

Albino, L. F. T. \& Barreto, S. R. L. T. (2003) Criação de codornas para produção de ovos e carne. Editora Aprenda Fácil, 268 p.

Barreto, S. L. D. T., Quirino, B. J. D. S., Brito, C. O., Umigi, R. T., Araujo, M. S. D., Rocha, T. C. D., \& Pereira, C. G. (2007). Efeitos de níveis nutricionais de energia sobre o desempenho e a qualidade de ovos de codornas européias na fase inicial de postura. Revista Brasileira de Zootecnia, 36(1), 86-93. https://doi.org/10.1590/S1516-35982007000100011.

Correa, G. S. S., Silva, M. A., Correa, A. B., Fontes, D. O., Torres, R. A., Dionello, N. J. L., Santos, G.G. \& Freitas, L. S. (2007) Exigência de proteína bruta e energia metabolizável para codornas de corte EV1. Arquivo Brasileiro de Medicina Veterinária e Zootecnia, 59(3), 797-804. https://doi.org/10.1590/S010209352007000300035

Drumond, E. S. C., Gonçalves, F. M., Veloso, R. D. C., Amaral, J. M., Balotin, L. V., Pires, A. V., \& Moreira, J. (2013) Curvas de crescimento para codornas de corte. Ciência Rural, 43(10), 1872-1877. https://doi.org/10.1590/S0103-84782013001000023

Finco, E. M., Marcato, S. M., Furlan, A. C., Rossi, R. M., Grieser, D. D. O., Zancanela, V., Oliveira, T. M. M. \& Stanquevis, C. E. (2016) Adjustment of four growth models through Bayesian inference on weight and body nutrient depositions in laying quail. Revista Brasileira de Zootecnia, 45(12), 737-744. https://doi.org/10.1590/s1806-92902016001200002

Firat, M. Z., Karaman, E., Başar, E. K., \& Narinc, D. (2016) Bayesian analysis for the comparison of nonlinear regression model parameters: an application to the growth of Japanese quail. Brazilian Journal of Poultry Science, 18.19-26. https://doi.org/10.1590/1806-9061-2015-0066

Grieser, D. D. O., Marcato, S. M., Furlan, A. C., Zancanela, V., Vesco, A. P. D., Batista, E., Ton, A. P. S. \& Perine, T. P. (2018) Estimation of growth parameters of body weight and body nutrient deposition in males and females of meat-and laying-type quail using the Gompertz model. Revista Brasileira de Zootecnia, 47. https://doi.org/10.1590/rbz4720170083

Hurtado-Nery, V. L., Torres-Novoa, D. M., \& Castro-Romero, A. S. (2014). Efecto de los niveles de energía metabolizable y proteína sobre el desempeño zootécnico de codornices en postura. Ciencia y Agricultura, 11(2), 9-16.

Mota, L. F. M, Alcantâra, D. C., Abreu, L. R. A., Costa, L. S., Pires, A. V., Bonafé, C. M., Silva, M. A., \& Pinheiro, S. R. F. (2015) Crescimento de codornas de diferentes grupos genéticos por meio de não lineares. Arquivo Brasileiro de Medicina Veterinária e Zootecnia, 67(5), 1372-1380. https://doi.org/10.1590/1678-4162-7534

Moura, G. D. S., Barreto, S. L. D. T., Donzele, J. L., Hosoda, L. R., Pena, G. D. M., \& Angelini, M. S. (2008) Dietas de diferentes densidades energéticas mantendo constante a relação energia metabolizável: nutrientes para codornas japonesas em postura. Revista Brasileira de Zootecnia, 37(9), 1628-1633. https://doi.org/10.1590/S1516-35982008000900015

Raji, A. O., Girgiri, A. Y., Alade, N. K., \& Jauro, S. A. (2015) Characteristics and proximate composition of Japanese quail (Coturnix Japonica) carcass in a semi arid area of Nigeria. Trakia Journal of Sciences, 2, 159-165. doi:10.15547/tjs.2015.02.008

Rajini, R.A.; Narahari, D. Asha Rajini, R., \& Narahari, D. (1998) Dietary energy and protein requirements of growing japanese quails in the tropics. Indian Journal of Animal Sciences, 68(10), 1082-1086.

Rocha-Silva, M., Araujo, C. V., Pires, A. V., Paula, E. J. H., Ferreira, E. B., \& Silva, F. G. (2016) Curva de crescimento de codornas de corte por meio de modelos de regressão não-lineares. Archives of Veterinary Science, 21(4). http://dx.doi.org/10.5380/avs.v21i4.41571

Rossi, R. M., \& Dos Santos, L. A. (2014) Modelagem Bayesiana para curvas de crescimentos de codornas assumindo assimetria nos erros. Semina: Ciências Agrárias, 35(3), 1637-1647. 10.5433/1679-0359.2014v35n3p1637

Rossi, R. M., De Oliveira Grieser, D., Almeida Conselvan, V., \& Marcato, S. M. (2017) Growth curves in meat-type and laying quail: a Bayesian perspective. Semina: Ciências Agrárias, 38(4), 2743-2754. 10.5433/1679-0359.2017v38n4Supl1p2743

Spiegelhalter, D. J., Best, N. G., Carlin, B. P., \& Van Der Linde, A. (2002) Bayesian measures of model complexity and fit. Journal of the royal statistical society: Series b (statistical methodology), 64(4), 583-639. https://doi.org/10.1111/1467-9868.00353

Vieira, D. V. G., Nascimento Oliveira, E. J., Souza, R. G., Bourdon, V. D. D. S., De Oliveira, T. R., Da Silva, K. E. C.,Cruz, J. S., Stivanin, T. E., Souza, T. A., Nascimento, C., Rodrigues, K. F., Vaz, R. G. M. V., Lima, M. R., Cavalcante, D. T. \& Costa, F. G. P. (2020) Mathematical models to predict the energy requirements of Japanese quails from 01-35 days age. Research, Society and Development, 9(8), e777986544-e777986544. https://doi.org/10.33448/rsdv9i8.6544

Ton, A. P. S., Furlan, A. C., Martins, E. N., Toledo, J. B., Scherer, C., \& Conti, A. C. M. (2011) Exigências de lisina digestível e de energia metabolizável para codornas de corte em crescimento. Revista Brasileira de Zootecnia, 40(3), 593-601. https://doi.org/10.1590/S1516-35982011000300018 